\title{
Berufstätigkeit und Rückkehr an den Arbeitsplatz bei stationär- psychiatrisch behandelten Patienten
}

\section{Psychiatric Inpatient Treatment and Return to Work}

Autoren

Lena Mernyi, Patricia Hölzle, Johannes Hamann

Institut

Klinik für Psychiatrie und Psychotherapie, Klinikum rechts

der Isar, Technische Universität München

Schlüsselwörter

Belastungen am Arbeitsplatz, Rückkehr an den Arbeitsplatz, psychische Erkrankungen

Keywords

return to work, mental disorders, work related stress

factors

\section{Bibliografie}

DOI https://doi.org/10.1055/s-0043-101901

Online-Publikation: 12.5.2017 | Psychiat Prax 2018; 45 :

197-205

(c) Georg Thieme Verlag KG Stuttgart · New York

ISSN 0303-4259

Korrespondenzadresse

Lena Mernyi, Klinik und Poliklinik für Psychiatrie und

Psychotherapie, Technische Universität München,

Möhlstraße 26, 81675 München

Lena.Mernyi@gmail.com

\section{ZUSAMMENFASSUNG}

Ziel der Studie Untersuchung der Beschäftigungsquote und des Wiedereinstiegs in den Beruf unter stationär-psychiatrisch behandelten Patienten.

Methodik Stichtagsvollerhebung stationär-psychiatrisch behandelter Patienten im Großraum München/Augsburg und anschließende Einzelinterviews.

Ergebnisse $21 \%$ der 815 untersuchten Patienten hatten ein festes Arbeitsverhältnis. Viele Patienten kehrten nicht wieder an ihren Arbeitsplatz zurück.

Schlussfolgerung Patienten sind nach Entlassung gefährdet, aus dem Arbeitsleben auszuscheiden. Deshalb, sollten arbeitsbezogene Belastungsfaktoren in der Behandlung frühzeitig thematisiert werden und Unterstützung beim Wiedereinstiegsprozess gewährleistet werden.

\section{ABSTRACT}

Objective People with mental diseases have a high risk of unemployment and they have only limited access to the labor market. The return to work is often associated with fears.

The present study aims to provide an overview of the number of hospitalized psychiatric patients with permanent employment. Moreover it should give an insight into the process of return to work, the experiences patients gain and the support they receive.

Methods In the participating clinics we measured the number of patients with permanent employment. The main inclusion criteria for further survey were the status of permanent employment and age between 18 and 65. The participating patients were interviewed on two occasions, at the time of inclusion and 3 months after the patient was discharged. The questions addressed working conditions, job satisfaction and the process of return-to-work. For statistical analysis, descriptive statistics (frequencies, means, standard deviations) were used.

Results Only $21 \%$ of $n=815$ inpatients of the participating hospitals were permanently employed. Many patients did not return to work after being discharged. In many cases the interviewed patients saw a connection between their job and their current episode of illness. In this context patients reported unsatisfying workplace conditions such as long working hours, bad work organization and social conflicts.

Conclusions For mentally ill patients, the employment rate in the primary labor market is devastating low. After psychiatric inpatient treatment patients are at high risk to lose their jobs. In order to prevent this development, work-related stress factors should be discussed with inpatients at an early stage and support should be provided during the return-to-work-process. 


\section{Einleitung}

In Deutschland stehen psychische Erkrankungen als Ursache für Arbeitsunfähigkeitszeiten mittlerweile an vierter Stelle der häufigsten Diagnosen. Zudem ist die durchschnittliche Krankheitsdauer bei psychischen Erkrankungen doppelt so lang wie bei anderen Erkrankungen [1]. Eine ebenso deutliche Zunahme ist bei den durch psychische Erkrankungen verursachten Erwerbsminderungsrenten zu verzeichnen [2]. Als Gründe für den Anstieg von psychischen Erkrankungen und der dadurch verursachten Arbeitsausfälle werden unter anderem die Enttabuisierung des Themas sowie die steigende psychische Belastung am Arbeitsplatz diskutiert [3].

Auf der anderen Seite leistet Erwerbstätigkeit einen wichtigen Beitrag zum psychischen Wohlbefinden durch finanzielle Absicherung, Tagesstrukturierung, gesellschaftliche Akzeptanz, Stärkung des Selbstbewusstseins und Möglichkeit für soziale Kontakte [4]. Arbeitslosigkeit dagegen führt häufig zu einer Verschlechterung des psychischen Gesundheitszustands, was die Wichtigkeit der Teilnahme am Arbeitsleben für psychisch erkrankte Menschen unterstreicht [5].

Schließlich sind Menschen mit psychischen Erkrankungen mit Stigmatisierung und Diskriminierung konfrontiert. Das macht sich auch im Berufsleben bemerkbar und führt bei den Betroffenen zu Problemen im Umgang mit ihrer Erkrankung [6]. Auch auf Arbeitgeberseite ist man durch die Zunahme psychisch erkrankter Mitarbeiter und den dadurch steigenden Kosten vor neue Herausforderungen gestellt. Eine mangelhafte Aufklärung über die Ursachen und Folgen psychischer Erkrankungen führt zu Unsicherheit im Umgang mit betreffenden Mitarbeitern. Hinzu kommt, dass Arbeitgeber vielfach schlecht über mögliche Unterstützungsmaßnahmen informiert sind [7].

Die erkennbar schwierigen Voraussetzungen für eine berufliche Wiedereingliederung psychisch Erkrankter machen eine gute Zusammenarbeit und Interaktion der beteiligten Akteure (Krankenkassen, Rentenversicherungen, Gesundheitswesen, Betriebe) dringend notwendig [8]. Etablierte Unterstützungsmaßnahmen (betriebliches Eingliederungsmanagement und stufenweise Wiedereingliederung) sollen den Übergang von der stationären oder ambulanten psychiatrischen Behandlung in das Berufsleben erleichtern.

Zu all diesen Aspekten liegen aktuell kaum Daten vor. Entsprechend war Ziel der vorliegenden Arbeit einerseits einen Überblick darüber zu geben, wie viele Patienten, die (teil)stationär psychiatrisch behandelt werden, in einem festen Arbeitsverhältnis stehen. Zum anderen sollte untersucht werden, wie Patienten den Zusammenhang zwischen psychischer Erkrankung und Arbeit sehen, wie diese Patienten bei der Rückkehr an den Arbeitsplatz unterstützt werden und wie sich der Wiedereinstieg praktisch gestaltet.

\section{Methoden}

\section{Studienablauf}

Im Rahmen des zweistufigen Studiendesigns wurde zunächst mithilfe eines Screenings erfasst, wie viele der Patienten, die sich an den jeweiligen Erhebungstagen in (teil-)stationärer Be- handlung in den teilnehmenden Krankenhäusern befanden, einen laufenden Arbeitsvertrag auf dem ersten Arbeitsmarkt hatten. Dabei wurde eine Vollerhebung aller stationär behandelten Patienten im Großraum München/Augsburg angestrebt.

Im zweiten Schritt wurden alle Patienten mit laufendem Arbeitsvertrag um ihre Teilnahme an einer Interviewbefragung gebeten. In der vorliegenden Untersuchung verwendeten wir folgende Definition für „laufenden Arbeitsvertrag“: Mehr als geringfügige, laufende Beschäftigung (Teil- oder Vollzeit) auf dem ersten Arbeitsmarkt bzw. mehr als geringfügige selbstständige Tätigkeit.

\section{Ein- und Ausschlusskriterien}

Die Erhebung der Daten erfolgte in psychiatrischen Kliniken im Großraum München/Augsburg (Isar-Amper-Klinikum-Nord/ -Ost/-Süd-West, Klinik für Psychiatrie und Psychotherapie, Technische Universität München, Klinikum Landsberg am Lech, Danuvius Klinik Pfaffenhofen, Bezirkskrankenhaus Augsburg) auf allen allgemeinpsychiatrischen Stationen und Tagkliniken (nicht erfasst wurden Patienten auf Suchtstationen und in der Gerontopsychiatrie). Im Rahmen des Screenings wurden alle Patienten, die sich am jeweiligen Erhebungstag in einer der Kliniken befanden, erfasst. Dabei wurde aus logistischen Gründen je Klinik ein eigener Stichtag im Zeitraum von März 2014 bis August 2014 festgelegt.

Einschlusskriterien für die Teilnahme an der Interviewuntersuchung waren ein Alter von 18 bis 65 Jahren und ein aktuelles Arbeitsverhältnis auf dem ersten Arbeitsmarkt (mehr als geringfügige Beschäftigung). Ausschlusskriterium waren mangelhafte Sprachkenntnisse.

\section{Erhobene Daten}

Im Rahmen des Screenings wurden Krankenhaus und Stationstyp dokumentiert (Tagklinik, geschlossen, offen), allgemeine Daten zum Patienten festgehalten (Alter, Geschlecht, Hauptdiagnose, Krankheitsschwere nach Clinical Global Impression, CGI [9]) und das Vorliegen eines laufenden Arbeitsvertrags geprüft (Arbeitsvertrag? ja/nein). Patienten wurden in die Studie aufgenommen, wenn die Einschlusskriterien für die Interviewbefragung erfüllt waren und das Einverständnis zur Teilnahme gegeben wurde. Die Teilnehmer wurden zu zwei Zeitpunkten (möglichst kurz VOR Entlassung und 3 Monate NACH Entlassung) mithilfe eines semistrukturierten Fragebogens befragt.

Der erste Fragebogen (vor Entlassung) erfasste zunächst allgemeine soziodemografische Daten des Patienten, Angaben zum Arbeitsplatz (Branche, Funktion, Arbeitszeit) und zur subjektiven Zufriedenheit am Arbeitsplatz (Wertschätzung, Einkommen, Arbeitszeit, Arbeitsorganisation) (zum Teil in Anlehnung an den Stressreport Deutschland 2012 [10]). Darauf folgten Fragen zu Belastungen/Konflikten am Arbeitsplatz, welche Rolle die Arbeit als Krankheitsauslöser hatte, inwiefern sich die Erkrankung am Arbeitsplatz gezeigt hatte, sowie Fragen zu Ängsten und Plänen hinsichtlich der Rückkehr an den Arbeitsplatz.

Zur zweiten Befragung wurden die Patienten 3 Monate nach der Entlassung aus der stationären Behandlung telefonisch in- 
terviewt. Hier wurde festgehalten, ob eine Rückkehr an den alten Arbeitsplatz erfolgt war, welche Maßnahmen zur Unterstützung bei der Rückkehr unternommen worden waren (BEM, stufenweise Wiedereingliederung, Rückkehrgespräch), wie gut die Unterstützung durch die Klinik/den Betrieb gewesen ist, welche Personen an dem Wiedereinstieg beteiligt waren und ob $\mathrm{Ar}$ beitsplatzanpassungen (Inhalte, Arbeitszeiten, Versetzung) vorgenommen worden waren. Weitere Fragen bezogen sich darauf, wie die Patienten mit ihrer Diagnose am Arbeitsplatz umgegangen waren.

\section{Statistik}

Im Vordergrund der Auswertung standen deskriptive Statistiken (Häufigkeiten, Mittelwerte, Standardabweichungen). Die Interviews wurden quantitativ ausgewertet, d.h. bei dichotomen/gestuften Antwortformaten wurden Häufigkeiten und Mittelwerte bestimmt. Bei offenen Antwortformaten wurden Kategorien gebildet und deren Häufigkeit dargestellt.

Gruppenvergleiche für dichotome/kategorische Variablen wurden mit Chi ${ }^{2}$-Tests, Gruppenvergleiche für stetige Variablen mit t-Tests vorgenommen.

\section{Ergebnisse}

\section{Screening}

Insgesamt wurden im Rahmen des Screenings $\mathrm{N}=815$ Patienten erfasst. Davon hatten $\mathrm{n}=174$ und damit $21 \%$ einen laufenden Arbeitsvertrag auf dem ersten Arbeitsmarkt ( $\triangleright$ Tab. 1).

Sowohl zwischen den teilnehmenden Kliniken als auch zwischen verschiedenen Stationstypen waren deutliche Unterschiede erkennbar. Während in den Tagkliniken die Mehrheit der Patienten (54\%) einen laufenden Arbeitsvertrag hatte, waren es auf den offenen Stationen deutlich weniger (24\%). Auf den beschützten Stationen hatten lediglich $13 \%$ einen festen Arbeitsplatz $\left(\right.$ Chi $\left.^{2}=58,9 ; \mathrm{p}<0,0001\right)$.

Patienten mit der Hauptdiagnose Depression (ICD 10 F32 oder F33) waren zu 34\% (109 Patienten von $n=319$ ) in einem festen Arbeitsverhältnis, Patienten mit bipolarer Störung (F31) zu $25 \%$ (12 Patienten von $\mathrm{n}=48$ ) und Patienten mit einer schizophrenen Erkrankung (F20 und F25) zu 9\% (25 Patienten von $\left.\mathrm{n}=247 ; \mathrm{Chi}^{2}=52,1 ; \mathrm{p}<0,0001\right)$.

Hinsichtlich Geschlecht und Alter unterschieden sich die Patienten mit laufendem Arbeitsvertrag nicht von denen ohne laufenden Arbeitsvertrag.

Patienten mit laufendem Arbeitsvertrag waren im Mittel (CGI-Wert $M=4,7$; SD =1,0) weniger krank als Patienten ohne laufenden Arbeitsvertrag (CGI-Wert $M=5,1 ; S D=1,0 ; t=-4,9$; $\mathrm{p}<0,0001)$.

\section{Interviewstudie - Befragung vor Entlassung}

Von den insgesamt 174 Patienten mit einem laufenden Arbeitsvertrag gaben 81 Patienten (47\%) ihr Einverständnis zur der Teilnahme an der Studie.

Dabei war der Anteil der teilnehmenden Frauen $(n=35)$ und Männer ( $n=46)$ weitgehend ausgeglichen. Die Befragten waren zwischen 19 und 63 Jahre alt $(M=42,8$ Jahre; $S D=11,3)$. Die soziodemografischen Daten der Teilnehmer sind der $>$ Tab. 2 zu
- Tab. 1 Übersicht der Ergebnisse aus den Screeningdaten.

\begin{tabular}{|c|c|c|c|}
\hline & $\begin{array}{l}\text { gesamt } \\
\text { Anzahl }\end{array}$ & $\begin{array}{l}\text { laufender } \\
\text { Arbeitsver- } \\
\text { trag }\end{array}$ & $\begin{array}{l}\text { kein laufen- } \\
\text { der Arbeits- } \\
\text { vertrag }\end{array}$ \\
\hline $\begin{array}{l}\text { erfasste Patienten } \\
\text { insgesamt }\end{array}$ & 815 & $174(21 \%)$ & $641(79 \%)$ \\
\hline \multicolumn{4}{|l|}{ Geschlecht } \\
\hline männlich & 345 & $76(22 \%)$ & $269(78 \%)$ \\
\hline weiblich & 470 & $98(21 \%)$ & $372(79 \%)$ \\
\hline Alter & & $\begin{array}{l}M=42,8 \\
S D=11,3\end{array}$ & $\begin{array}{l}M=43,7 \\
S D=15,4\end{array}$ \\
\hline CGI & & $\begin{array}{l}M=4,7 \\
S D=1,0\end{array}$ & $\begin{array}{l}M=5,1 \\
S D=1,0\end{array}$ \\
\hline \multicolumn{4}{|l|}{ Stationstyp } \\
\hline offene Station & 399 & $94(24 \%)$ & $305(76 \%)$ \\
\hline geschlossene Station & 349 & $44(13 \%)$ & $305(87 \%)$ \\
\hline Tagklinik & 67 & $36(54 \%)$ & $31(46 \%)$ \\
\hline \multicolumn{4}{|l|}{$\begin{array}{l}\text { Hauptdiagnose } \\
\text { (nach ICD-10) }\end{array}$} \\
\hline F0 & 17 & $3(18 \%)$ & $14(82 \%)$ \\
\hline $\mathrm{F} 1$ & 20 & $3(15 \%)$ & $17(85 \%)$ \\
\hline $\mathrm{F} 2$ & 272 & $25(9 \%)$ & $247(91 \%)$ \\
\hline F31 & 48 & $12(25 \%)$ & $36(75 \%)$ \\
\hline $\mathrm{F} 32 / 33 / 34$ & 319 & $109(34 \%)$ & $210(66 \%)$ \\
\hline $\mathrm{F} 4$ & 59 & $15(25 \%)$ & $44(74 \%)$ \\
\hline F5 & 1 & $0(0 \%)$ & $1(100 \%)$ \\
\hline F6 & 67 & $7(10 \%)$ & $60(90 \%)$ \\
\hline sonstige & 12 & $0(0 \%)$ & $12(100 \%)$ \\
\hline
\end{tabular}

entnehmen. Hinsichtlich der für alle Patienten beim Screening erhobenen Maße (Alter, Geschlecht, Diagnose und CGI) zeigten sich keine signifikanten Unterschiede zwischen Patienten, die ihr Einverständnis zum Interview gaben und denen, die dies verweigerten.

Im Hinblick auf die Branchen war ein Großteil der Patienten im Dienstleistungssektor (63\%) tätig, 17\% war in der Industriebranche, $10 \%$ im Gesundheitswesen, $6 \%$ im Bildungswesen und $4 \%$ im Finanzwesen. Im Mittel hatten die Patienten vor 71 Tagen $(S D=58,4)$ zuletzt gearbeitet und waren bereits seit 11,3 Jahren $(S D=9,8)$ an ihrem Arbeitsplatz beschäftigt. Nur wenige Patienten (3\%) befanden sich noch in der Probezeit.

Von den 81 befragten Patienten war die Mehrheit (88\%) in einem festen Anstellungsverhältnis, $6 \%$ der Patienten war verbeamtet und $3 \%$ waren jeweils befristet angestellt oder selbstständig. Eine nicht unerhebliche Anzahl der Befragten, 24\%, arbeitete im Schichtdienst.

Die offizielle Wochenarbeitszeit betrug im Durchschnitt 35,1 Stunden $(S D=8,4)$. Dabei variierte sie zwischen 10 und 
Tab. 2 Soziodemografische Daten der Studienteilnehmer.

\begin{tabular}{|c|c|c|}
\hline & $\mathrm{n}=\mathbf{8 1}$ & $\%$ \\
\hline \multicolumn{3}{|l|}{ Geschlecht } \\
\hline männlich & 46 & 57 \\
\hline weiblich & 35 & 43 \\
\hline \multicolumn{3}{|l|}{ Diagnose } \\
\hline F2 & 11 & 14 \\
\hline F31 & 6 & 7 \\
\hline F32/F33/F34 & 51 & 63 \\
\hline F4 & 5 & 7 \\
\hline F6 & 7 & 9 \\
\hline \multicolumn{3}{|l|}{ Muttersprache } \\
\hline deutsch & 65 & 80 \\
\hline andere & 16 & 20 \\
\hline \multicolumn{3}{|l|}{ Familienstand } \\
\hline ledig & 38 & 47 \\
\hline verheiratet & 33 & 41 \\
\hline geschieden & 10 & 12 \\
\hline \multicolumn{3}{|l|}{ Wohnsituation } \\
\hline alleine & 39 & 48 \\
\hline Familie/Partner & 32 & 40 \\
\hline Wohngemeinschaft & 2 & 3 \\
\hline sonstiges & 8 & 10 \\
\hline \multicolumn{3}{|l|}{ Schulabschluss } \\
\hline Hauptschule & 25 & 31 \\
\hline polytechnische Oberschule & 1 & 1 \\
\hline mittlere Reife & 23 & 28 \\
\hline Abitur & 27 & 33 \\
\hline Schulabschluss im Ausland erworben & 5 & 6 \\
\hline \multicolumn{3}{|l|}{ Berufsausbildung } \\
\hline ja & 67 & 83 \\
\hline nein & 14 & 17 \\
\hline \multicolumn{3}{|l|}{ abgeschlossenes Studium } \\
\hline ja & 20 & 25 \\
\hline nein & 61 & 75 \\
\hline
\end{tabular}

55 Stunden. Im Gegensatz dazu lagen die Angaben zu den tatsächlichen Wochenarbeitsstunden im Mittel bei 39,7 Stunden (SD =11,1), wobei die Spanne von 12 bis 70 Stunden reichte.

- Tab. 3 gibt einen Überblick über die subjektive Zufriedenheit der Patienten an ihrem Arbeitsplatz. Dabei fällt auf, dass sich ein Großteil der Patienten den Anforderungen an ihre fachlichen Kenntnisse gewachsen fühlte, während fast die Hälfte
- Tab. 3 Angaben zur subjektiven Zufriedenheit in der Arbeit, bezogen auf die letzten 12 Monate vor Ausbruch der Krankheitsepisode.

$$
\mathbf{n}=\mathbf{8 1} \quad \%
$$

Wie häufig hatten Sie das Gefühl, dass die Tätigkeit wichtig ist?

\begin{tabular}{|l|r|r|}
\hline häufig & 59 & 73 \\
\hline manchmal & 13 & 16 \\
\hline selten & 8 & 10 \\
\hline nie & 1 & 1 \\
\hline
\end{tabular}

Hatten Sie das Gefühl, ausreichend für Ihre Tätigkeit wertgeschätzt zu werden?

\begin{tabular}{|l|l|l|}
\hline ausreichend & 32 & 40 \\
\hline teils/teils & 30 & 37 \\
\hline nicht ausreichend & 19 & 24 \\
\hline
\end{tabular}

\section{Wie zufrieden waren Sie mit dem Einkommen?}

\begin{tabular}{|l|c|c|}
\hline sehr zufrieden & 16 & 20 \\
\hline zufrieden & 41 & 51 \\
\hline weniger zufrieden & 18 & 22 \\
\hline nicht zufrieden & 6 & 7 \\
\hline
\end{tabular}

\section{Wie zufrieden waren Sie mit den Arbeitszeiten?}

\begin{tabular}{|l|r|r|}
\hline sehr zufrieden & 17 & 21 \\
\hline zufrieden & 38 & 47 \\
\hline weniger zufrieden & 23 & 28 \\
\hline nicht zufrieden & 3 & 4 \\
\hline
\end{tabular}

Wie zufrieden waren Sie mit der Arbeitsorganisation (Aufgabenverteilung, interne Kommunikation bei Aufgaben)?

\begin{tabular}{|l|r|r|}
\hline sehr zufrieden & 15 & 19 \\
\hline zufrieden & 34 & 42 \\
\hline weniger zufrieden & 26 & 32 \\
\hline nicht zufrieden & 6 & 7 \\
\hline
\end{tabular}

Fühlten Sie sich den Anforderungen an Ihre fachlichen Kenntnisse und Fertigkeiten in der Regel gewachsen, eher überfordert oder eher unterfordert?

\begin{tabular}{|l|l|l|}
\hline den Anforderungen gewachsen & 54 & 67 \\
\hline eher überfordert & 16 & 20 \\
\hline eher unterfordert & 11 & 14 \\
\hline
\end{tabular}

Fühlten Sie sich den Anforderungen durch die Arbeitsmenge bzw. das Arbeitspensum in der Regel gewachsen, eher überfordert oder eher unterfordert?

\begin{tabular}{|l|c|c|}
\hline den Anforderungen gewachsen & 35 & 43 \\
\hline eher überfordert & 39 & 48 \\
\hline eher unterfordert & 7 & 9 \\
\hline Gab es zwischenmenschliche Konflikte am Arbeitsplatz? \\
\hline ja & 40 & 49 \\
\hline nein & 41 & 51 \\
\hline
\end{tabular}


- Tab. 3 (Fortsetzung)

\begin{tabular}{|l|c|c|}
\hline \multicolumn{1}{|c|}{$\mathbf{n}=\mathbf{8 1}$} & $\%$ \\
\hline Wie zufrieden waren Sie insgesamt mit & Ihrer Arbeit? & \\
\hline sehr zufrieden & 16 & 20 \\
\hline zufrieden & 42 & 52 \\
\hline weniger zufrieden & 19 & 24 \\
\hline nicht zufrieden & 4 & 5 \\
\hline
\end{tabular}

bezüglich des Arbeitspensums eher überfordert war. 37\% der Patienten fühlten sich nur teilweise für ihre Tätigkeit wertgeschätzt und $24 \%$ fühlten sich nicht ausreichend wertgeschätzt.

Beinahe die Hälfte der Befragten berichtete über zwischenmenschliche Konflikte am Arbeitsplatz. Am häufigsten lagen Konflikte mit Arbeitskollegen (80\%) vor. Von Konflikten mit Vorgesetzten berichteten $46 \%$ der Teilnehmer, seltener (18\%) gab es Konflikte mit Kunden (Mehrfachantworten).

Für 38 Patienten (47\%) spielte die Arbeit eine große oder sehr große Rolle bei der Entstehung der Krankheitsepisode.

Die Erkrankung machte sich am Arbeitsplatz bei den Patienten auf unterschiedliche Weise bemerkbar. 32 Patienten (40\%) schilderten ein Nachlassen der Konzentration während der Arbeit („Fehler gemacht“, „häufige Unterbrechungen der Arbeit“, „langsamer gearbeitet als sonst“ usw.). Bei 13 Personen (16\%) spielte Müdigkeit und eine verminderte Leistungsfähigkeit eine Rolle. Jeweils 8 Personen (10\%) gaben an, in der Arbeit geweint zu haben bzw. Angst- und Panikattacken gehabt zu haben. Acht Personen (10\%) waren regelmäßig zu spät zur Arbeit gekommen, 7 Personen ( $9 \%$ ) berichteten von sozialem Rückzug, 4 Personen (5\%) zeigten aggressives Verhalten und 3 Personen (4\%) konsumierten Alkohol während der Arbeitszeit.

In $>$ Tab. 4 sind Angaben der Patienten zu den Erwartungen und Ängsten hinsichtlich der Rückkehr an den Arbeitsplatz zu sehen. Dabei gaben $42 \%$ der Patienten an, eher schlecht bis sehr schlecht auf den Wiedereinstieg in den Beruf vorbereitet zu sein. 38\% der befragten Patienten fühlte sich durch die Angst vor Verlust des Arbeitsplatzes unter Druck gesetzt, möglichst bald wieder an den alten Arbeitsplatz zurückzukehren. In Hinblick auf die Rückkehr an den Arbeitsplatz hatten viele Patienten (32\%) Angst vor der Reaktion der Arbeitskollegen.

\section{Interviewbefragung - 3 Monate nach Entlassung}

Die Durchführung der zweiten Befragung war bei 48 Patienten (59\%) der insgesamt 81 teilnehmenden Patienten möglich ( $\triangleright$ Tab.5). Unter den telefonisch erreichbaren Personen waren 34 (71\%) an ihren alten Arbeitsplatz zurückgekehrt. Von den 14 Personen (29\%), die nicht zurückgekehrten waren, hatten 3 (6\%) eine neue Stelle angenommen, 5 Personen (10\%) waren weiterhin bzw. erneut im Krankenstand, bei 1 Person (2\%) war der Wiedereinstieg abgebrochen worden und 3 (6\%) waren auf der Suche nach einem neuen Arbeitsplatz. Die Hälfte der Patienten war im Rahmen einer stufenweisen Wiedereingliederung an den Arbeitsplatz zurückgekehrt.
- Tab. 4 Angaben zu den Erwartungen an den Wiedereinstieg in den Beruf.

\begin{tabular}{|c|c|c|}
\hline & $n=81$ & $\%$ \\
\hline \multicolumn{3}{|c|}{$\begin{array}{l}\text { Wie stark sind/wären die Ängste in Bezug auf die Rückkehr an der } \\
\text { Arbeitsplatz? }\end{array}$} \\
\hline sehr stark & 18 & 22 \\
\hline stark & 17 & 21 \\
\hline mittelmäßig & 22 & 27 \\
\hline kaum vorhanden & 14 & 17 \\
\hline nicht vorhanden & 10 & 12 \\
\hline
\end{tabular}

Welche Bereiche machen Ihnen Sorgen hinsichtlich der Rückkehr an den Arbeitsplatz? (offene Frage, Auswertung der häufigsten Antworten)

\begin{tabular}{|l|c|c|}
\hline Reaktion der Kollegen & 26 & 35 \\
\hline Arbeit nicht mehr zu schaffen & 22 & 29 \\
\hline erneut krank zu werden & 13 & 17 \\
\hline $\begin{array}{l}\text { als weniger belastbar eingeschätzt zu } \\
\text { werden }\end{array}$ & 8 & 11 \\
\hline \begin{tabular}{l} 
Arbeit zu verlieren \\
\hline
\end{tabular} & 6 & 8 \\
\hline
\end{tabular}

Hat bei Ihnen früher schon einmal ein Wiedereinstieg in den Beruf nach längerer Arbeitsunfähigkeit stattgefunden?$$
\text { ja }
$$

nein

\begin{tabular}{|l|l|}
\hline 21 & 26 \\
\hline 60 & 74 \\
\hline
\end{tabular}

Würden Sie sagen, dass Sie in Ihrem Wunschberuf gearbeitet haben oder hätten Sie lieber in einem anderen Beruf gearbeitet?

\begin{tabular}{|l|c|c|}
\hline ich arbeite im Wunschberuf & 56 & 69 \\
\hline $\begin{array}{l}\text { ich hätte gerne in einem anderen Beruf } \\
\text { gearbeitet }\end{array}$ & 23 & 28 \\
\hline fehlende Antwort & 2 & 3 \\
\hline Haben Sie vor, an Ihren Arbeitsplatz zurückzukehren? & \\
\hline ja & 73 & 90 \\
\hline nein & 1 & 1 \\
\hline vielleicht & 7 & 9 \\
\hline
\end{tabular}

Manche Patienten fühlen sich von äußeren Faktoren, wie etwa wegen Angst vor Verlust des Arbeitsplatzes oder aus finanziellen Gründen sehr stark unter Druck gesetzt, möglichst bald an den Arbeitsplatz zurückzukehren. Wie stark ist dieser Druck von außen bei Ihnen?

\begin{tabular}{|l|c|c|}
\hline sehr stark & 11 & 14 \\
\hline stark & 20 & 25 \\
\hline mittelmäßig & 15 & 19 \\
\hline kaum vorhanden & 17 & 21 \\
\hline nicht vorhanden & 18 & 22 \\
\hline
\end{tabular}

Durch welche Faktoren fühlen Sie sich dabei besonders unter Druck gesetzt? (offene Frage)

finanzielle Gründe

21

26 
Tab. 4 (Fortsetzung)

\begin{tabular}{|l|c|c|}
\hline & $\mathbf{n = 8 1}$ & $\%$ \\
\hline Angst vor Verlust des Arbeitsplatzes & 8 & 10 \\
\hline beide Faktoren & 23 & 28 \\
\hline keine Angabe & 29 & 36 \\
\hline Wie gut fühlen Sie sich auf die Rückkehr vorbereitet? & \\
\hline sehr gut & 4 & 5 \\
\hline gut & 25 & 31 \\
\hline mittel & 18 & 22 \\
\hline eher schlecht & 15 & 19 \\
\hline sehr schlecht & 19 & 24 \\
\hline
\end{tabular}

- Tab.5 Befragung der Patienten nach der Entlassung aus der Klinik.

\begin{tabular}{|c|c|c|}
\hline & $n=48$ & $\%$ \\
\hline \multicolumn{3}{|c|}{ Sind Sie wieder an Ihren Arbeitsplatz zurückgekehrt? } \\
\hline ja & 34 & 71 \\
\hline nein & 14 & 29 \\
\hline fehlende Werte & 0 & 0 \\
\hline \multicolumn{3}{|c|}{$\begin{array}{l}\text { Sind Sie regulär an den Arbeitsplatz zurückgekehrt oder im } \\
\text { Rahmen einer stufenweise Wiedereingliederung? }\end{array}$} \\
\hline stufenweise Wiedereingliederung & 19 & 40 \\
\hline reguläre Rückkehr & 19 & 40 \\
\hline fehlende Werte & 10 & 21 \\
\hline \multicolumn{3}{|c|}{$\begin{array}{l}\text { Wurde Ihnen ein Betriebliches Eingliederungsmanagement } \\
\text { angeboten? }\end{array}$} \\
\hline ja & 13 & 27 \\
\hline nein & 34 & 71 \\
\hline fehlende Werte & 1 & 2 \\
\hline \multicolumn{3}{|c|}{$\begin{array}{l}\text { Hat ein offizielles Rückkehrgespräch mit lhrem Vorgesetzten } \\
\text { stattgefunden? }\end{array}$} \\
\hline ja & 18 & 38 \\
\hline nein & 20 & 42 \\
\hline fehlende Werte & 10 & 21 \\
\hline \multicolumn{3}{|c|}{ Wie gut hat Sie die Klinik beim Wiedereinstieg unterstützt? } \\
\hline sehrgut & 11 & 23 \\
\hline gut & 10 & 21 \\
\hline mittel & 15 & 31 \\
\hline eher schlecht & 3 & 6 \\
\hline sehr schlecht & 3 & 6 \\
\hline fehlende Werte & 6 & 13 \\
\hline
\end{tabular}

- Tab. 5 (Fortsetzung)

\begin{tabular}{|l|l|l}
\hline & $n=48$ & $\%$ \\
\hline
\end{tabular}

Wie gut hat Sie Ihr Betrieb/Ihr Unternehmen beim Wiedereinstieg unterstützt?

\begin{tabular}{|l|c|c|}
\hline sehr gut & 12 & 25 \\
\hline gut & 15 & 31 \\
\hline mittel & 9 & 19 \\
\hline eher schlecht & 4 & 8 \\
\hline sehr schlecht & 1 & 2 \\
\hline fehlende Werte & 7 & 15 \\
\hline
\end{tabular}

Am Wiedereinstieg beteiligte Personen im Betrieb (neben dem Arbeitgeber)

\begin{tabular}{|l|r|r|}
\hline Personalrat & 8 & 17 \\
\hline Betriebsarzt & 6 & 13 \\
\hline Schwerbehindertenvertretung & 3 & 6 \\
\hline Sozialpädagoge & 1 & 2 \\
\hline fehlende Werte & 30 & 63 \\
\hline
\end{tabular}

Wurden langfristige Arbeitsplatzanpassungen vorgenommen? Wie etwa in Bezug auf...

\begin{tabular}{|l|r|r|}
\hline Arbeitszeit & 6 & 13 \\
\hline Inhalte & 5 & 10 \\
\hline Versetzung in anderen Bereich & 5 & 10 \\
\hline keine & 18 & 38 \\
\hline fehlende Werte & 14 & 29 \\
\hline
\end{tabular}

Was haben Sie Ihrem Vorgesetzten als Grund für Ihre Fehlzeit genannt?

\begin{tabular}{|l|c|c|}
\hline richtige Diagnose & 32 & 67 \\
\hline „psychisches Problem“ & 5 & 10 \\
\hline körperliche Erkrankung & 2 & 4 \\
\hline nichts genannt („war krank“) & 5 & 10 \\
\hline fehlende Werte & 4 & 9 \\
\hline
\end{tabular}

Was haben Sie Ihren Kollegen als Grund für Ihre Fehlzeit genannt?

\begin{tabular}{|l|r|r|}
\hline richtige Diagnose & 21 & 44 \\
\hline „psychisches Problem“ & 2 & 4 \\
\hline körperliche Erkrankung & 2 & 4 \\
\hline nichts genannt („war krank“) & 16 & 33 \\
\hline fehlende Werte & 7 & 15 \\
\hline
\end{tabular}

Die Vorbereitung durch die Klinik auf den beruflichen Wiedereinstieg beurteilten $50 \%$ der Patienten als gut bis sehr gut. Während des stationären Aufenthalts hatten die Patienten am häufigsten (65\%) mit ihren behandelnden Ärzten über das Thema Arbeit gesprochen. Zweithäufigster Ansprechpartner (48\%) waren die Sozialarbeiter. Insgesamt gaben die Patienten in $45 \%$ der Fälle an, über die Möglichkeit einer stufenweisen Wieder- 
eingliederung und rechtliche Aspekte gesprochen zu haben, 13 \% hatten über arbeitsplatzbezogene Belastungen gesprochen und wie sie bei Verschlechterung der Symptome am Arbeitsplatz reagieren sollen. $4 \%$ hatten Hilfestellung bei der Kontaktaufnahme mit dem Arbeitgeber erhalten und $4 \%$ waren beraten worden, eine neue Stelle anzunehmen.

$66 \%$ der Patienten fühlten sich beim Wiedereinstieg in den Beruf sehr gut bis gut von ihrem Betrieb unterstützt. Während der Fehlzeit hatte mehr als die Hälfte der Patienten (66\%) Kontakt zu ihren Vorgesetzen gehalten, 33 \% hatten Kontakt zu Kollegen gehabt. Ein Großteil der Patienten, nämlich 67\%, hatte den Arbeitgeber über die Diagnose informiert. Mit den Arbeitskollegen sprachen nur $48 \%$ offen über die Diagnose, $42 \%$ nannte gegenüber den Kollegen keinen genauen Grund für die Fehlzeit.

Ein Rückkehrgespräch mit dem Vorgesetzten hatte nur bei $38 \%$ der Patienten stattgefunden.

Nur wenige Patienten (27\%) erhielten eine Einladung zu einem betrieblichen Eingliederungsmanagement (BEM). Davon nahmen 39\% der Patienten das Angebot an.

Auf die Frage, welche zusätzlichen Unterstützungsmaßnahmen sich die Patienten gewünscht hätten, beklagten viele Patienten den schwierigen Übergang von der stationären Behandlung zurück in das Berufsleben. Gerade in der Anfangsphase des Wiedereinstiegs habe ein Ansprechpartner gefehlt. Von einigen Patienten wurde kritisiert, dass kein Rückkehrgespräch mit dem Arbeitgeber stattgefunden hatte.

\section{Diskussion}

Die vorliegende Erhebung zeigt, dass bei Patienten psychiatrischer Kliniken nur ein geringer Anteil noch auf dem ersten Arbeitsmarkt beschäftigt ist. Nach einem Klinikaufenthalt gelingt nur zwei Drittel der Patienten mit festem Arbeitsplatz der Wiedereinstieg innerhalb von 3 Monaten nach Entlassung. Viele Patienten sehen einen Zusammenhang zwischen ihrer Arbeit und der Entstehung der Krankheitsepisode. Häufig genannte Belastungsfaktoren am Arbeitsplatz waren zwischenmenschliche Konflikte und Überforderung mit der Arbeitsmenge. Hinsichtlich der Vorbereitung der Rückkehr an den Arbeitsplatz bestehen sowohl aus Sicht der Patienten als auch im Hinblick auf die Umsetzung strukturierter Maßnahmen (z. B. stufenweise Wiedereingliederung) Verbesserungsmöglichkeiten.

Zunächst scheint die Anzahl der an den Arbeitsplatz zurückgekehrten Patienten mit $71 \%$ relativ hoch zu sein. Berücksichtigt man jedoch den insgesamt geringen Anteil an Berufstätigen unter den stationär psychiatrisch behandelten Patienten, erscheint ein (zumindest vorübergehendes) Ausscheiden aus dem Arbeitsleben von fast $30 \%$ alarmierend hoch.

Zugleich muss beachtet werden, dass lediglich die Hälfte aller berufstätigen Patienten zu einer Teilnahme an der Befragung bereit waren. Es ist also möglich, dass gerade Patienten, bei denen ein Wiedereinstieg in den Beruf weniger wahrscheinlich war, nicht miterfasst wurden.

Man muss also davon ausgehen, dass es nach jeder einzelnen stationär-psychiatrischen Behandlung einer beträchtlichen Anzahl an berufstätigen Patienten nicht gelingt, wieder auf dem
Arbeitsmarkt Fuß zu fassen. Dies ist vor dem Hintergrund eines damit verbundenen sozialen Abstiegs bzw. der dann eingeschränkten Teilhabe am gesellschaftlichen Leben insgesamt besorgniserregend [11, 12].

Entsprechend betrachten wir zunächst mögliche Gründe für dieses Muster, die sich aus unseren Ergebnissen andeuten, und diskutieren jeweils mögliche Maßnahmen zur Verbesserung des Übergangs von der Klinik in die Arbeitswelt.

\section{Arbeit als Auslöser, Schwierigkeiten am Arbeitsplatz}

Viele Patienten hielten ihre Arbeitssituation für einen (Mit-) Auslöser ihrer aktuellen Krankheitsepisode. Aus den Einschätzungen ragen vor allem negative Bewertungen der Arbeitszeit und Arbeitsorganisation mit vor allem mengenmäßiger Überforderung heraus. Diese Beobachtung deckt sich mit Ergebnissen aus anderen Untersuchungen, wonach Arbeitsbedingungen wie hohe Anforderungen und Überstunden die Entstehung von Depression begünstigen können [13].

Zudem berichten viele Patienten über zwischenmenschliche Konflikte am Arbeitsplatz. Hierbei ist allerdings zu beachten, dass zwischenmenschliche Konflikte am Arbeitsplatz ein insgesamt häufiges Phänomen darstellen [10, 14].

Im Rahmen unserer Untersuchung ist dabei nicht zu klären, ob diese Umstände Auslöser der Erkrankung sind oder vielmehr schon als Ausdruck einer ggf. noch subklinischen Symptomatik zu werden sind. Hilfreich zur Verhinderung einer ungünstigen Interaktion zwischen psychischer Erkrankung und Arbeit wären hier Interventionen im Sinne einer Sekundär- bzw. Tertiärprävention [15, 16]. Aufseiten der Patienten wäre das Erkennen von Frühwarnzeichen und adäquate Reaktion darauf sinnvoll, aufseiten der Arbeitgeber und Vorgesetzten frühzeitiges Erkennen und Ansprechen von Änderungen der Arbeitsleistung. Ersteres ist bereits Bestandteil psychoedukativer Programme und eines strukturierten Entlassmanagements, könnte allerdings noch für mehr Patienten implementiert werden [17]. Spezifische Programme für Führungskräfte oder Personaler zielen bereits heute schon auf das Früherkennen von möglichen psychischen Erkrankungen ab [18], sind aber ebenfalls noch nicht in der Breite implementiert.

\section{Angst vor der Rückkehr und Vorbereitung des Wiedereinstiegs}

Die Rückkehr an den Arbeitsplatz ist für viele Patienten angstbesetzt, was sich mit Ergebnissen aus anderen Untersuchungen deckt [19]. Die Ängste liegen zum einen an den schlechten Erfahrungen vor dem Krankenhausaufenthalt, zum anderen an der Unsicherheit, wie man von Kollegen und Vorgesetzten empfangen wird bzw. ob die geforderten Leistungen wieder erbracht werden können.

In diesen Bereichen kann unserer Ansicht nach das psychiatrische Hilfesystem z.B. im Rahmen der stationären Versorgung eine wichtige Rolle in der Vorbereitung der Patienten spielen. Kliniken sollten Konzepte erarbeiten, in denen für die ja wenigen betroffenen Patienten das Thema Arbeit regelhaft fokussiert wird und entsprechende Ressourcen zur gezielten Unterstützung am Übergang zwischen stationärer Behandlung und Wiedereinstieg in die Arbeit ausgebaut werden. 
Dabei sollten vor allem angstbesetzte Themen aus dem Kontext Arbeit thematisiert und angegangen werden. Im Hinblick auf die Leistungsfähigkeit am Arbeitsplatz sollte die aktuelle Leistungsfähigkeit im Rahmen der Arbeitstherapie bzw. Ergotherapie und ggf. mithilfe einer neuropsychologischen Diagnostik möglichst valide eingeschätzt werden [20]. Im Falle von Leistungseinschränkungen muss dies entweder therapeutisch angegangen oder den Patienten angeboten werden, unter Hinzuziehung des Arbeitgebers z. B. zeitlich begrenzte Arbeitsanpassungen vorzunehmen [21].

Da in unserer Befragung auffiel, dass sich ein Großteil der Patienten den fachlichen Anforderungen gewachsen fühlte, während sich viele hinsichtlich der Arbeitsmenge eher überfordert fühlten, könnten Arbeitsanpassungen sich häufig in reduzierten Arbeitszeiten niederschlagen. Vor allem auch in Bezug auf letzteren Punkt ist die Rolle des Sozialdienstes bei der Unterstützung und Beratung der Patienten zu stärken.

Auffallend viele Patienten berichteten über zwischenmenschliche Konflikte am Arbeitsplatz, die zum Teil auch Sorgen im Hinblick auf den Wiedereinstieg bereiteten. Dass Konflikte und Spannungen mit Vorgesetzten und Kollegen neben den Arbeitsbedingungen einen entscheidenden Faktor bei der Entstehung von arbeitsplatzbezogenen Ängsten spielen, zeigten bereits Untersuchungen von Bürger und Koch [22, 23].

Es scheint damit ein relevantes Thema zu sein, das bei der Vorbereitung auf den Wiedereinstieg mehr berücksichtigt werden sollte. Hölzle et al. haben ein Psychoedukationsprogramm zum Wiedereinstieg in die Arbeit untersucht, in dem u.a. das Rückkehrgespräch mit Vorgesetzten und Kollegen thematisiert und im Rahmen von Rollenspielen eingeübt wird (Psychiatrische Praxis, in Vorbereitung).

Schließlich fällt in unseren Daten auf, dass strukturierte Ansätze des Wiedereinstiegs in die Arbeit (z. B. stufenweise Wiedereingliederung, betriebliches Eingliederungsmanagement, offizielle „Rückkehrgespräche“) nach wie vor nicht regelhaft umgesetzt werden. Damit wird gleichzeitig auch die Möglichkeit von Arbeitsanpassungen verpasst. Ursächlich für diesen Umstand sind wahrscheinlich beide Seiten: Arbeitgeber, indem Instrumente wie BEM nicht regelhaft angeboten werden, Patienten, indem sie derartige Angebote häufig nicht anstoßen oder sogar ablehnen [24].

Das seltene Angebot vom BEM ist womöglich auch dem Umstand geschuldet, dass die Wiedereingliederung von psychisch erkrankten Arbeitnehmern von den Unternehmern als besonders schwierig empfunden wird und häufig ein Verbesserungsbedarf beim betrieblichen Eingliederungsmanagement betreffender Mitarbeiter gesehen wird [25].

\section{Stärken und Schwächen der Arbeit}

Als Stärke unserer Untersuchung sind der Versuch einer Vollerhebung und das große untersuchte Versorgungsgebiet zu sehen. Damit ist es wahrscheinlich, dass unsere Ergebnisse nah an der Versorgungsrealität sind.

Die Studie ist dadurch limitiert, dass viele Patienten, die zur Teilnahme infrage kamen, nicht in die Befragung eingewilligt haben und ein beträchtlicher Teil nicht mehr für die Follow-upUntersuchung erreichbar war. Dadurch kommt es möglicher- weise zu einer Verzerrung der Ergebnisse in eine zu positive Richtung. Eine weitere Schwäche der Studie ist, dass ausschließlich Angaben von Patientenseite erhoben wurden und die Sicht der Arbeitgeber keinen Niederschlag fand.

\section{Fazit}

Zusammenfassend zeigen unsere Ergebnisse, dass berufstätige Patienten nach einer stationär-psychiatrischen Behandlung in vielen Fällen nicht an ihren Arbeitsplatz zurückkehren.

Die Gründe dafür scheinen, genau wie die arbeitsbezogenen Gegebenheiten sehr unterschiedlich zu sein, was eine individuelle Unterstützung und Vorbereitung auf den Wiedereinstieg in den Beruf erforderlich macht.

Dennoch geben unsere Ergebnisse Hinweise auf einige Faktoren (Konflikte, große Arbeitsmenge), die häufig im Zusammenhang mit psychischen Belastungen am Arbeitsplatz zu beobachten sind und die daher bei der Vorbereitung auf den Wiedereinstieg besonders beachtet werden sollten.

\section{Danksagung}

Die Autoren danken den folgenden Kliniken für ihre Unterstützung des Projektes: Isar-Amper-Klinikum-München Ost (Prof. Dr. Albus, Dr. Pfeiffer, Dr. Schleuning, Dr. Schwarz), Lech-Mangfall-Klinik Landsberg am Lech (Dr. Kuhlmann), Danuvius Klinik Pfaffenhofen (PD Dr. Messer), Bezirkskrankenhaus Augsburg (Prof. Dr. Schmauß).

\section{KONSEQUENZEN FÜR KLINIK UND PRAXIS}

- Da die Arbeitsverhältnisse von Patienten nach einem psychiatrischen Krankenhausaufenthalt gefährdet sind, ist dem Wiedereingliederungsprozess besondere Aufmerksamkeit zu widmen.

- Im Rahmen der stationären Versorgung sollten angstbesetzte Themen aus dem Kontext Arbeit thematisiert und angegangen werden.

- Die aktuelle Leistungsfähigkeit sollte im Rahmen einer Arbeitstherapie und ggf. mithilfe einer neuropsychologischen Diagnostik möglichst valide eingeschätzt und ggf. Gegenstand therapeutischer Angebote sein.

Interessenkonflikt

Johannes Hamann erhielt in den vergangenen 3 Jahren Vortragshonorare und Forschungsbeihilfe von Janssen Cilag, Ely Lilly und Otsuka.

Literatur

[1] Badura BDA, Schröder H, Klose J, Meyer M, Hrsg. Fehlzeitenreport 2015: Neue Wege für mehr Gesundheit - Qualitätsstandards für ein zielgruppenspezifisches Gesundheitsmanagement. Berlin: Springer; 2015

[2] Deutsche Rentenversicherung. Statistik der Deutschen Rentenversicherung: Indikatoren zu Erwerbsminderungsrente im Zeitablauf 2015. Berlin: Deutsche Rentenversicherung; 2015 
[3] Richter D, Berger K. Are mental disorders increasing? Update of a systematic review on repeated cross-sectional studies. Psychiat Prax 2013; 40: $176-182$

[4] Ulrich E, Wülser M. Gesundheitsmanagement in Unternehmen: Arbeitspsychologische Perspektiven. Wiesbaden: Springer Gabler; 2015

[5] Paul KI, Moser K. Unemployment impairs mental health: Meta-analyses. Journal of Vocational Behavior 2009; 74: 264-282

[6] Bezborodovs N, Thornicroft G. Stigmatisation of mental illness in the workplace: evidence and consequence. Die Psychiatrie - Grundlagen und Perspektiven 2013; 10: $102-107$

[7] Freigang-Bauer I, Gröben F. Eingliederung von Mitarbeitern mit psychischen Erkrankungen. Düsseldorf: Hans-Böckler-Stiftung; 2011

[8] Brieger P, Hoffmann H. How can the mentally ill achieve sustained employment? Supported employment versus pre-vocational training. Nervenarzt 2012; 83: $840-846$

[9] Busner ], Targum SD. The Clinical Global Impressions Scale: Applying a Research Tool in Clinical Practice. Psychiatry (Edgmont) 2007; 4: $28-37$

[10] Lohmann-Haislah A. Stressreport Deutschland 2012. Psychische Anforderungen, Ressourcen und Befinden. Dortmund: Bundesanstalt für Arbeitsschutz und Arbeitsmedizin; 2012

[11] Warr PB. Work, unemployment, and mental health. Oxford: Oxford University Press; 1987

[12] Provencher HL, Gregg R, Mead S et al. The role of work in the recovery of persons with psychiatric disabilities. Psychiatr Rehabil J 2002; 26: $132-144$

[13] Stuke H, Bermpohl F. Which Working Conditions Promote the Development of Depressive Disorders? Psychiat Prax 2016; 43: 245 - 252

[14] Bundesanstalt für Arbeitsschutz und Arbeitsmedizin. Wenn aus Kollegen Feinde werden: Der Ratgeber zum Umgang mit Mobbing. Dortmund: Bundesanstalt für Arbeitsschutz und Arbeitsmedizin; 2011
[15] Berger M, Gravert C, Schneller C et al. Prävention und Behandlung psychischer Störungen am Arbeitsplatz. Der Nervenarzt 2013; 84: $1291-1298$

[16] Mendel R, Kissling W, Hamann J. Vom Tabu zum Kostenfaktor Warum die Psyche plötzlich ein Thema für Unternehmen ist. Wirtschaftspsychologie aktuell 2010; 2: $23-27$

[17] Steffen S, Kösters M, Becker T. Discharge planning in mental health care: A systematic review of the recent literature. Acta Psych Scand 2009; 120: 1 -9

[18] Hamann J, Mendel R, Reichhart T et al. A “Mental-Health-at-theWorkplace” Educational Workshop Reduces Managers' Stigma Toward Depression. The Journal of nervous and mental disease 2016; 204: $61-63$

[19] Linden M, Muschalla B. Anxiety disorders and workplace-related anxieties. Journal of Anxiety Disorders 2007; 21: 467 -474

[20] Plohmann AM. Relevanz neuropsychologischer Gutachten zur Beurteilung der Arbeitsfähigkeit und Integrität. Epileptologie 2008; 25: $182-190$

[21] Baer N. Probleme bei Arbeitsplatzerhalt und Wiedereingliederung Grundlagen und Interventionsmöglichkeiten in der Behandlung. PSYCH up2date 2015; 9: 369 - 386

[22] Bürger W, Koch U. Arbeitsbelastungen und ihre Bedeutung für Patienten zu Beginn von stationärer psychosomatischer Fachbehandlung. Z Med Psychol 1995; 2: 66 - 78

[23] Koch U, Laschinsky D. Ein Fragebogen zur Erfassung der Situation am Arbeitsplatz und in der Familie (KOLA). Psychol Prax 1979; 4: $165-173$

[24] Gröben F, Freigang-Bauer I, Barthen L. Betriebliches Eingliederungsmanagement von Mitarbeitern mit psychischen Störungen. Prävention und Gesundheitsförderung 2011; 6: 229-237

[25] Fulton S, Clohesy D, Wise FM et al. A goal-directed woodwork group for men in community rehabilitation - A pilot project. Australian occupational therapy journal 2016; 63: 29-36 